\title{
Risk Factors for Cerebrovascular Disease as Correlates of Cognitive Function in a Stroke-Free Cohort
}

\author{
David W. Desmond, PhD; Thomas K. Tatemichi, MD; Myunghee Paik, PhD; Yaakov Stern, PhD
}

- We investigated the relationship between risk factors for cerebrovascular disease and cognitive function in 249 stroke-free community volunteers (age, 70.8 \pm 6.7 years; education, $12.3 \pm 4.6$ years) who were given tests of memory, language, visuospatial, abstract reasoning, and attentional skills. Using logistic regression analyses, we examined hypertension, diabetes mellitus, myocardial infarction, angina, hypercholesterolemia, and cigarette smoking as potential correlates of performance within these cognitive domains. Controlling for demographic factors within the logistic models, diabetes mellitus was a significant independent correlate of abstract reasoning deficits (odds ratio, $10.9 ; 95 \%$ confidence interval, 2.2 to 54.9 ) and visuospatial dysfunction (odds ratio, 3.5; confidence interval, 1.2 to 10.7), while hypercholesterolemia was a significant independent correlate of memory dysfunction (odds ratio, 3.0; confidence interval, 1.4 to 6.6). Prolonged exposure to vascular risk factors such as diabetes mellitus and hypercholesterolemia may lead to atherosclerotic disease, possibly resulting in "silent" infarctions or impaired cerebral blood flow and a decline in cognitive functioning.

(Arch Neurol. 1993;50:162-166)

$\mathbf{R}$ isk factors for stroke have been extensively examined and are well known; these include hypertension, diabetes mellitus, cardiac disease, and smoking. ${ }^{1-3}$ Elevated lipid levels have also been associated with stroke, ${ }^{4}$ and small artery occlusion by cholesterol emboli has been reported..$^{5-7}$ Targeted interventions to modify risk factors, especially hypertension, have proved effective in reducing stroke risk. ${ }^{8}$ Cerebrovascular risk factors have also been examined in healthy, asymptomatic subjects as determinants of cognitive function to identify the earliest neurologic consequences of risk factor exposure. ${ }^{9}$ If stroke-free elderly subjects with vascular risk factors exhibit deficits in intellectual performance, important public health implications arise. Cognitive decline, possibly mediated through

\footnotetext{
Accepted for publication August 3, 1992.

From the Departments of Neurology (Drs Desmond, Tatemichi, and Stern) and Psychiatry (Dr Stern), School of Public Health (Dr Paik), and Gertrude H. Sergievsky Center (Dr Stern), Columbia University, College of Physicians and Surgeons, New York, NY.

Presented in part at the international Conference on Stroke, Geneva, Switzerland, May 1991.

Reprint requests to the Neurological Institute, $710 \mathrm{~W} 168$ th St, New York, NY 10032 (Dr Tatemichi).
}

the mechanism of clinically silent stroke, may be the first sign of significant cerebrovascular disease.

Previous studies have suggested that certain risk factors for cerebrovascular disease, including hypertension, diabetes mellitus, cardiac disease, and hypercholesterolemia, can affect performance on a variety of cognitive tasks.? However, these studies have generally examined single risk factors in small samples without controlling for a broad range of potentially confounding exposures that might also affect cognition. The purpose of our study was to investigate the independent effects of cerebrovascular risk factors on specific aspects of cognition while adjusting for confounding exposures in a large cohort of elderly stroke-free subjects.

\section{SUBJECTS AND METHODS Subjects}

As part of a longitudinal study of stroke and dementia, ${ }^{10}$ we recruited 249 subjects (mean age, $70.8 \pm 6.7$ years; mean education, $12.3 \pm 4.6$ years) free of cerebrovascular disease by history or neurological examination. The majority of these subjects were randomly selected from Medicare records and a commercial list $(53.0 \%)$, and the remainder of the cohort included volunteers from the community $(29.7 \%)$ and spouses of stroke patients $(17.3 \%)$.

\section{Procedures}

Each subject received neurological, neuropsychological, and functional examinations. Medical and risk factor histories were collected through subject self-report by interview with a physician. The primary risk factors considered in this study included history of hypertension, diabetes mellitus, myocardial infarction, angina, hypercholesterolemia, and consistent cigarette use (ie, history of smoking at least one cigarette each day for a period of at least 1 year). Other risk factors were not investigated due to lack of association with stroke risk, rarity of exposure, or unacceptably high levels of missing information. We also collected information relevant to other potential correlates of cognitive function, including demographic factors; medication use; consistent alcohol use (ie, history of consumption of at least one serving of beer, wine, or hard liquor each week for a period of at least 6 months); and depression, assessed with the Hamilton Rating Scale for Depression. ${ }^{11}$

\section{Reliability and Validity of Medical History Information}

The reliability and validity of the medical history self-reports were evaluated. The medical history interview was readministered to 39 randomly selected members of the overall cohort. Each 
of the risk factors being investigated in this study had been endorsed by at least 10 members of this smaller sample, and 10 subjects with no history of exposure to these risk factors were also interviewed. Permission was sought from the subjects to contact their personal physicians to validate their self-reports, with laboratory data being collected and used when available. Standard values used in our institution served as criteria for diabetes mellitus (ie, fasting glucose $\geq 7.7 \mathrm{mmol} / \mathrm{L}[\geq 140 \mathrm{mg} / \mathrm{dL}]$ ) and hypercholesterolemia (ie, total cholesterol $\geq 6.20 \mathrm{mmol} / \mathrm{L}[\geq 240$ $\mathrm{mg} / \mathrm{dL}]$ ). Electrocardiographic results were used to diagnose myocardial infarction. The diagnosis of hypertension required multiple blood pressure measurements with elevated values (ie, systolic blood pressure $\geq 160 \mathrm{~mm} \mathrm{Hg}$ or diastolic blood pressure $\geq 95 \mathrm{~mm} \mathrm{Hg}$ ) taken on separate days; the complexity of this diagnosis and the extensive chart review that was required necessitated our reliance on the physician's diagnosis in almost all cases.

\section{Neuropsychological Evaluation}

Neuropsychological testing was performed in either English or Spanish, based on the language spoken in the subject's home. The test battery ${ }^{12}$ included measures of memory function (the Selective Reminding Test and a multiple-choice recognition version of the Benton Visual Retention Test), abstract reasoning skills (the Similarities subtest of the Wechsler Adult Intelligence ScaleRevised), verbal functions (selected items from the Boston Naming Test and selected subtests from the Boston Diagnostic Aphasia Examination), visuospatial functions (multiple-choice matching version of the Benton Visual Retention Test and the Rosen figure drawing test), and attentional skills (timed targetfinding task).

\section{Statistical Analyses}

Neuropsychology subtest scores were converted into $z$ scores, and the $z$ scores for subtests within each cognitive domain were averaged to represent overall performance within that domain. Each subtest was equally weighted in these transformations. The distribution of scores within each cognitive domain was divided at the mean (ie, $z=0$ ) to form two groups, representing "better" and "worse" performance. The mean was selected as the cutoff because a more stringent criterion (eg, 2 SDs below the mean) could mask potentially subtle risk factor effects. Subjects were grouped based on the position of their scores in each of the distributions and not on external criteria because no other normative database exists for this battery of neuropsychological tests.

Logistic regression analyses were then performed to determine the correlates of these performance-based groupings for each cognitive domain. Logistic regression techniques were selected as the primary analytic method rather than multiple linear regression because the beta coefficient may be transformed into an odds ratio $(O R)$ for each independent variable, providing clearer information regarding the magnitude of risk factor effects. Following the logistic analyses, however, we performed multiple regression analyses to determine whether comparable results would be obtained if the dependent variables were treated as continuous, rather than categorical, data. Independent variables for all analyses included vascular risk factors (ie, hypertension, diabetes mellitus, myocardial infarction, angina, hypercholesterolemia, and consistent cigarette use), demographic variables (ie, age, education, race, sex, and occupation), medications used (ie, pharmacologic treatment for hypertension, diabetes, cardiac disease, and hyperlipidemia), total score on the Hamilton Depression Rating Scale, and consistent alcohol use. Demographic variables were forced into each of the final equations, with age and education being entered as continuous data and race, sex, and occupation being entered as categorical data.

\section{RESULTS}

Descriptive statistics and frequencies of vascular risk factor exposure and medication use are presented in Table

\begin{tabular}{|lcc|}
\hline \multicolumn{2}{|c|}{$\begin{array}{c}\text { Table 1.Descriptive Statistics and Frequencies } \\
\text { of Risk Factor Exposure and Medication Use }\end{array}$} \\
\hline \multicolumn{1}{|c|}{ Variable } & $\mathbf{n}$ & $\%$ of Cohort \\
\hline Demographic & & \\
Sex, male & 85 & 34.1 \\
Race, white & 130 & 52.2 \\
Risk factors & & \\
Hypertension & 105 & 42.2 \\
Diabetes mellitus & 29 & 11.6 \\
Myocardial infarction & 19 & 7.7 \\
Angina & 34 & 13.7 \\
Hypercholesterolemia & 62 & 25.2 \\
Consistent alcohol use & 127 & 51.0 \\
Consistent cigarette use & 121 & 48.6 \\
Medication treatment & & \\
Hypertension & 84 & 33.7 \\
Diabetes mellitus & 18 & 7.2 \\
Cardiac disease & 53 & 21.3 \\
Hyperlipidemia & 13 & 5.2 \\
\hline
\end{tabular}

1. Women outnumbered men in this elderly cohort; this is consistent with population trends related to the greater life expectancy of women. ${ }^{13}$ The most frequently acknowledged risk factor was hypertension, followed by hypercholesterolemia, cardiac disease, and diabetes mellitus. The results of the reliability and validity analyses of the medical history self-reports are presented in Table 2 . Reliability coefficients (ie, $\mathrm{ks}$ ) were highest for self-reports regarding diabetes mellitus, myocardial infarction, and angina, while positive predictive value was highest for hypercholesterolemia, myocardial infarction, and angina.

\section{Vascular Risk Factors as Correlates of Cognitive Function}

The results of the logistic regression analyses are discussed with regard to each cognitive domain.

Memory Functions.-Significant correlates of memory dysfunction included older age (OR, 1.1; 95\% confidence interval $[\mathrm{CI}], 1.0$ to 1.2$)$, lower education (OR, 1.2; CI, 1.1 to 1.4 ), nonwhite race (OR, 2.7; CI, 1.3 to 5.5), male sex (OR, 4.9; CI, 2.2 to 10.7), and nonprofessional occupation (OR, 2.7; CI, 1.2 to 6.4). Controlling for these factors within the multivariate model, hypercholesterolemia (OR, 3.0; CI, 1.4 to 6.6) was a significant independent correlate of memory dysfunction.

Descriptive statistics and neuropsychological test results broken down by history of hypercholesterolemia are presented in Table 3.

Abstract Reasoning.--Lower education (OR, 1.4; CI, 1.2 to 1.6 ) and nonwhite race (OR, 7.4; CI, 3.5 to 15.8) were significant correlates of abstract reasoning deficits. Age, sex, and occupation were forced into the final model. Controlling for these factors within the multivariate model, diabetes mellitus (OR, 10.9; CI, 2.2 to 54.9) was a significant independent correlate of abstract reasoning deficits.

Descriptive statistics and neuropsychological test results broken down by history of diabetes mellitus are also shown in Table 3.

Verbal Functions.-Lower education (OR, 1.2; CI, 1.1 to 1.3), male sex (OR, 3.2; CI, 1.6 to 6.6), and nonwhite race (OR, 3.1; CI, 1.6 to 6.3) were significant correlates of verbal dysfunction. Age and occupation were forced into the final model. None of the vascular risk factors were significantly related to performance in this cognitive domain.

Visuospatial Functions.-Lower education (OR, 1.2; CI, 
Table 2,-Reliability and Validity of Risk Factor History*

\begin{tabular}{|lcccc|}
\hline \multicolumn{1}{|c|}{ Risk Factor } & Reliability, $\mathbf{~}$ & Positive & Specificity & Predictive Value \\
\hline Hypertension & .80 & .90 & .79 & .81 \\
Diabetes mellitus & .94 & 1.00 & .93 & .81 \\
Myocardial infarction & .93 & .90 & .96 & .96 \\
Angina & .86 & .64 & .90 & .98 \\
Hypercholesterolemia & .57 & .68 & .94 & .92 \\
\hline
\end{tabular}

*Results are based on data collected from a randomly selected subgroup of 39 subjects.

Table 3.-Descriptive Statistics and Test Results by History of Hypercholesterolemia and Diabetes Mellitus*

\begin{tabular}{|c|c|c|c|c|}
\hline \multirow[b]{2}{*}{ Variable } & \multicolumn{2}{|c|}{ Hypercholesterolemia } & \multicolumn{2}{|c|}{ Diabetes Mellitus } \\
\hline & $\begin{array}{c}\text { Yes } \\
(n=62)\end{array}$ & $\begin{array}{c}\text { No } \\
(n=187)\end{array}$ & $\begin{array}{c}\text { Yes } \\
(\mathbf{n}=29)\end{array}$ & $\begin{array}{c}\text { No } \\
(n=220)\end{array}$ \\
\hline Age & $71.6 \pm 6.8$ & $70.6 \pm 6.6$ & $69.8 \pm 6.3$ & $71.0 \pm 6.8$ \\
\hline Education & $13.2 \pm 4.5$ & $12.0 \pm 4.5$ & $10.2 \pm 5.1$ & $12.6 \pm 4.4$ \\
\hline Memory & $-0.03 \pm 0.81$ & $0.02 \pm 0.87$ & $-0.23 \pm 0.82$ & $0.03 \pm 0.86$ \\
\hline Abstraction & $0.23 \pm 0.81$ & $-0.08 \pm 0.87$ & $-0.57 \pm 0.91$ & $0.07 \pm 0.82$ \\
\hline Verbal & $0.11 \pm 0.77$ & $-0.03 \pm 0.73$ & $-0.24 \pm 0.63$ & $0.03 \pm 0.76$ \\
\hline Visuospatial & $0.16 \pm 0.85$ & $-0.06 \pm 0.84$ & $-0.35 \pm 0.63$ & $0.05 \pm 0.86$ \\
\hline Attention & $0.09 \pm 0.63$ & $-0.03 \pm 0.72$ & $-0.05 \pm 0.47$ & $0.01 \pm 0.72$ \\
\hline HDRS & $3.8 \pm 4.4$ & $3.4 \pm 3.8$ & $3.5 \pm 3.9$ & $3.5 \pm 4.0$ \\
\hline
\end{tabular}

*Results presented for each cognitive domain are $z$ scores based on the performance of the full cohort. Values are expressed as means \pm SDs. HDRS indicates Hamilton Depression Rating Scale.

1.1 to 1.3 ), nonwhite race (OR, $4.6 ; \mathrm{CI}, 2.3$ to 9.1 ), and nonprofessional occupation (OR, 2.3; $\mathrm{CI}, 1.0$ to 5.0 ) were significant correlates of visuospatial dysfunction. Age and sex were forced into the final model. Controlling for these factors within the multivariate model, diabetes mellitus (OR, $3.5 ; \mathrm{CI}, 1.2$ to 10.7$)$ was a significant independent correlate of visuospatial dysfunction.

Attentional Functions. - Lower education (OR, 1.2; CI, 1.1 to 1.3 ) and male sex (OR, 2.2; CI, 1.2 to 4.3 ) were significant correlates of attentional deficits. Age, race, and occupation were forced into the final equation. None of the vascular risk factors were significantly related to performance in this cognitive domain.

The results of the multiple regression analyses were comparable with those of the logistic regression analyses, with hypercholesterolemia being a significant independent correlate of memory dysfunction $(\beta=-.14)$ and diabetes mellitus being a significant independent correlate of abstract reasoning deficits $(\beta=-.11)$. A significant relationship was not found between diabetes mellitus and visuospatial dysfunction, however. As noted in the logistic analyses, none of the vascular risk factors were significantly related to performance in the verbal or attentional domains.

\section{COMMENT}

Specific risk factors for cerebrovascular disease were associated with cognitive dysfunction in our stroke-free elderly cohort. Logistic regression analyses determined that diabetes mellitus was strongly related to abstract reasoning deficits and visuospatial dysfunction, while hypercholesterolemia was strongly related to memory dysfunction. Multiple linear regression analyses supported all of these findings except the relationship between diabetes mellitus and visuospatial dysfunction; thus, this finding should be considered tentative. These significant relationships were independent of other variables that may influence cognitive function, such as demographic factors. In addition, medication use, a history of consistent alcohol use, and total score on the Hamilton Rating Scale for Depression were not significantly related to performance within any of the cognitive domains.

The frequency of exposure to vascular risk factors in our cohort was consistent with prevalence rates reported in the general population. ${ }^{14}$ Although we did not measure vascular risks directly, subject self-reports of medical and risk factor history were both reliable, with coefficients showing good to excellent agreement beyond chance, ${ }^{15}$ and valid, with high positive predictive value and good to excellent sensitivity and specificity. The reliability of self-reported risk factor history has been examined in another study in the same community using a random sample of 145 subjects. ${ }^{16}$ Reliability coefficients for self-reported history of hypertension $(\kappa, .69)$, diabetes mellitus $(\kappa, .60)$, and cholesterol level $(\kappa, .60)$ were all significant $(P<.001)$, representing good agreement beyond chance. In our sample, disagreement between patient's self-report of hypercholesterolemia and physician's records occurred in nine of 39 cases. In eight of those nine cases the disagreements resulted from false-negative reports by the patient. To the extent that underenumeration of exposure status might have occurred in the cohort overall, the relationship between hypercholesterolemia and memory function should be even more robust.

Patients with diabetes mellitus may exhibit deficits in general neuropsychological performance ${ }^{17}$ as well as in specific areas of cognition, such as memory. ${ }^{18}$ Although depression may contribute to some of the cognitive deficits 
exhibited by diabetics, ${ }^{19,20}$ the degree of cognitive impairment appears to be related, more importantly, to glycemic control, ${ }^{21}$ with significant cognitive deficits in acute hypoglycemia ${ }^{22}$ and milder deficits in hyperglycemic states. ${ }^{23}$ In addition, patients with more severe diabetes, defined as a longer duration of illness, greater incidence of complications, or increased frequency of hypoglycemic episodes, exhibit greater cognitive deficits. ${ }^{24}$

Several studies have suggested that hypercholesterolemia and hypertriglyceridemia are associated with impaired cognitive function. Better performance in mental status testing may be related to higher levels of highdensity lipoprotein cholesterol ${ }_{r}^{25}$ and a form of dementia due to extremely high levels of plasma lipids has been reported ${ }^{26}$ Lowering of the total serum cholesterol level may improve cognitive performance. ${ }^{27}$ Similarly, elevated triglyceride levels have been associated with deficits in coding, attention, and reaction time testing. ${ }^{28}$ Treatment with gemfibrozil, resulting in a reduced triglyceride but not total cholesterol level, has led to improvement in mental status. $^{29}$

Patients with ischemic heart disease tend to be more cognitively impaired than those with nonischemic disease, particularly in the areas of memory and concentration. ${ }^{30}$ Prospective cardiac transplantation patients exhibit deficits in multiple cognitive domains, ${ }^{30}$ while patients with valvular heart disease may have memory impairment. ${ }^{31}$

Hypertension has been associated with deficits in general intellectual performance,,$^{32}$ memory function, ${ }^{33}$ visuospatial function, ${ }^{34}$ and performance in simple ${ }^{34}$ and choice $^{35}$ reaction time testing. In contrast to these significant findings in small cohorts of hypertensive subjects, we found no relationship between hypertension and cognition. Similarly, the Framingham study found only a weak relationship between "definite hypertension" and immediate verbal memory and attention in 2032 elderly strokefree subjects. After adjusting for multiple comparisons, this finding was not significant. No relationship was obtained between any cognitive function and systolic or diastolic blood pressure, borderline hypertension, or the use of antihypertensive medications. ${ }^{36}$

One possible explanation for our findings is clinically "silent" infarction resulting from exposure to risk factors. Elevated levels of plasma lipids and diabetes mellitus have been associated with microcirculatory disturbances; microangiopathy, sometimes resulting in lacunar infarction; and, in extreme cases, "subcortical arteriosclerotic encephalopathy." ${ }^{37}$ In an imaging study of 246 neurologically normal adults, silent lacunar infarctions were noted in $13 \%$, with male sex, older age, history of hypertension, mean arterial blood pressure, and retinal artery sclerosis significantly related to the lesions. ${ }^{38}$ In the Framingham cohort, glucose intolerance was the only significant correlate of imaged but clinically silent lesions in patients with first stroke. ${ }^{39}$ In other imaging studies of subjects with ${ }^{40}$ and without ${ }^{41}$ symptoms of cerebrovascular disease, a significant relationship has been noted between number of risk factors and number of "silent" or incidental infarctions, but the role of individual risk factors in producing such lesions was not investigated.

Vascular risk factors also may have transient effects on cognitive function by producing disorders of cerebral blood flow. Reductions in cerebral perfusion have been observed in combination with elevated cholesterol and triglyceride levels, either due to large-vessel atherosclero- $\mathrm{sis}^{42}$ or impaired vasomotor reactivity with increased small-vessel resistance. ${ }^{43}$ Diabetes mellitus also affects cerebral blood flow and may produce disorders of autoregulation and reactivity. ${ }^{44}$ The increased blood viscosity of diabetics ${ }^{45}$ may contribute to reduced cerebral blood flow. ${ }^{46}$ Increased viscosity is also seen in patients with angina, possibly due to an elevated hematocrit, ${ }^{47}$ providing another basis for reduced flow. ${ }^{46,48}$ Cerebral blood flow also has been found to be reduced in patients with clinically silent infarctions. ${ }^{38}$

Although we documented adequate reliability and validity of subject self-reports regarding medical history, future studies should measure these factors directly. For example, plasma glucose level at the time of examination is related to cognitive performance. ${ }^{21} \mathrm{~A}$ similar relationship may apply to cholesterol, or its components. Should such dynamic relationships be demonstrated, it would suggest that metabolic factors or cerebral blood flow alterations affect cognition, rather than "silent" infarction. The most direct way to address these issues would be to examine clinically stroke-free subjects with brain imaging and cerebral blood flow studies along with neuropsychological testing.

The results of this study demonstrate an independent relationship between specific risk factors and cognition in individuals free of stroke. These findings support more aggressive management of vascular risk factors in strokefree populations, not only to reduce the incidence of stroke, but also to prevent subtle cognitive dysfunction that may result from the effects of those risk factors.

This work was supported in part by Grants R01-NS26179 and P01AG07232 from the National Institutes of Health, Bethesda, Md.

\section{References}

1. Wolf PA, D'Agostino RB, Kannel WB, Bonita R, Belanger AJ. Cigarette smoking as a risk factor for stroke. JAMA. 1988;259:1025-1029.

2. Wolf PA, D'Agostino RB, Belanger AJ, Kannel WB. Probability of stroke: a risk profile from the Framingham study. Stroke. 1991;22:312-318.

3. Mortel KF, Meyer JS, Sims PA, McClintic K. Diabetes mellitus as a risk factor for stroke. South Med J. 1990;83:904-911.

4. Wolf PA, D'Agostino RB, Belanger Al, Kelly-Hayes M, Kase CS, Kannel WB. Are blood lipids risk factors for stroke? Stroke. 1991;22:150. Abstract.

5. Beal MF, Williams RS, Richardson EP, Fisher CM. Cholesterol embolism as a cause of transient ischemic attacks and cerebral infarction. Neurology. 1981;31:860-865.

6. Buge A, Vincent D, Rancurel G, Baudrimont M, Dubas F, Hauw Jf. Embolies rétiniennes, musculaires et cutanées de cholestérol encéphalopathie progressive. Rev Neurol. 1985;141:578-582.

7. Laloux P, Brucher JM. Lacunar infarctions due to cholesterol emboli. Stroke. 1991;22:1440-1444.

8. SHEP Cooperative Research Group. Prevention of stroke by antihypertensive drug treatment in older persons with isolated systolic hypertension. JAMA. 1991;265:3255-3264.

9. Bornstein RA, Kelly MP. Risk factors for stroke and neuropsychological performance. In: Bornstein RA, Brown GG, eds. Neurobehavioral Aspects of Cerebrovascular Disease. New York, NY: Oxford University Press Inc; 1991; 10:182-201.

10. Tatemichi TK, Desmond DW, Mayeux R, et al. Dementia after stroke: baseline frequency, risks, and clinical features in a hospitalized cohort. Neurology. 1992;42:1185-1193.

11. Williams JBW. A structured interview guide for the Hamilton Depression Rating Scale. Arch Gen Psychiatry. 1988;45:742-747.

12. Stern $Y$, Andrews $H$, Pittman l, et al. Diagnosis of dementia in a heterogeneous population: development of a neuropsychological paradigmbased diagnosis of dementia and quantified correction for the effects of education. Arch Neurol. 1992;49:453-460.

13. US Dept of Commerce, Bureau of the Census. Projections of the population of the United States by age, sex, and race: 1983 to 2080 . Current Population Reports. Washington, DC: US Dept of Commerce; 1984. Series P-25; No. 952.

14. National Center for Health Statistics. Data from the National Health Survey. Vital Health Stat. 1990;10:84.

15. Fleiss JL. Statistical Methods for Rates and Proportions. 2nd ed. New York, NY: John Wiley \& Sons; 1981; 13:218. 
16. Shea S, Stein AD, Lantigua R, Basch CE. Reliability of the Behavioral Risk Factor Survey in a triethnic population. Am / Epidemiol. 1991;133:489500 .

17. Baade LE. Neuropsychological test differences between insulin dependent diabetic adults and controls. J Clin Exp Neuropsychol. 1988;10:89. Abstract.

18. Bale R. Brain damage in diabetes mellitus. Br J Psychiatry. 1973;122: 337-341.

19. Popkin M, Callies A, Lentz R, Colon E, Sutherland D. Prevalence of major depression, simple phobia, and other psychiatric disorders in patients with long-standing type i diabetes mellitus. Arch Gen Psychiatry. 1988;45: 64-68.

20. Tun PA, Perlmuter LC, Russo P, Nathan DM. Memory self-assessment and performance in aged diabetics and non-diabetics. Exp Aging Res. 1987; 13:151-157.

21. Holmes CS. Neuropsychological profiles in men with insulindependent diabetes. I Consult Clin Psychol. 1986;54:386-389.

22. Pramming $S$, Thorsteinsson B, Theilgaard A, Pinner EM, Binder C. Cognitive function during hypoglycemia in type I diabetes mellitus. $B M$ ). 1986;292:647-650.

23. Holmes CS, Hayford IT, Gonzalez IL, Weydert JA. A survey of cognitive functioning at different glucose levels in diabetic persons. Diabetes Care. 1983;6:180-185.

24. Skenazy JA, Bigler ED. Neuropsychological findings in diabetes mellitus. I Clin Psychol. 1984;40:246-258.

25. Postiglione A, Cortese C, Fischetti A, et al. Plasma lipids and geriatric assessment in a very aged population of South Italy. Atherosclerosis. 1989; 80:63-68.

26. Heilman K, Fisher WR. Hyperlipidemic dementia. Arch Neurol. $1974 ; 31: 67-68$.

27. Reitan RM, Shipley RE. The relationship of serum cholesterol changes to psychological abilities. J Gerontol. 1963;18:350-357.

28. Perlmuter LC, Nathan DM, Goldfinger SH, Russo PA, Yates J, Larkin $M$. Triglyceride levels affect cognitive function in noninsulin-dependent diabetics. / Diabetic Complications. 1988;2:210-213.

29. Rogers RL, Meyer JS, McClintic K, Mortel KF. Reducing hypertriglyceridemia in elderly patients with cerebrovascular disease stabilizes or improves cognition and cerebral perfusion. Angiology. 1989;40:260-269.

30. Bornstein R, Hammer D, Starling R, Stang J, Lewis R, Magorien R. Neuropsychological impairment in candidates for cardiac transplantation. In: Willner AE, Rodewald G, eds. Impact of Cardiac Surgery on the Quality of Life: Neurological and Psychological Aspects. 3rd ed. Orlando, Fla: Plenum Press: 1989:231-235.

31. Bethune DW. The assessment of organic brain damage following open heart surgery. In: Speidel H, Rodewaid G, eds. Psychic and Neurological Dysfunction After Open Heart Surgery. Stuttgart, Germany: George Thieme Verlag; 1980:100-106.
32. Schultz NR, Dineen IT, Elias MF, Pentz CA, Wood WG. WAIS performance for different age groups of hypertensive and control subjects during administration of a diuretic. J Gerontol. 1979;34:246-253.

33. Francheschi M, Tancredi O, Smirne S, Mercinelli A, Canal N. Cognitive processes in hypertension. Hypertension. 1982;4:226-229.

34. Boller F, Vrtunski B, Mack JL, Kim Y. Neuropsychological correlates of hypertension. Arch Neurol. 1977;34:701-705.

35. Light KC. Slowing of response time in young and middle-aged hypertensive patients. Exp Aging Res. 1975;1:209-227.

36. Farmer ME, White LR, Abbott RD, et al. Blood pressure and cognitive performance: the Framingham study. Am / Epidemiol. 1987;126:11031114.

37. Schneider $\mathrm{R}$, Klesewetter $\mathrm{H}$. The significance of microcirculatory disturbances in the pathogenesis of vascular dementia. Pharmacopsychiatry. $1988 ; 21: 11-16$

38. Kobayashi S, Okada K, Yamashita K. Incidence of silent lacunar lesion in normal adults and its relation to cerebral blood flow and risk factors. Stroke. $1991: 22: 1379-1383$

39. Kase CS, Wolf PA, Chodosh EH, et al. Prevalence of silent stroke in patients presenting with initial stroke: the Framingham study. Stroke. 1989; 20:850-852.

40. Awad IA, Spetzler RF, Hodak JA, Awad CA, Williams F, Carey R. Incidental lesions on magnetic resonance imaging of the brain: prevalence and clinical significance in various age groups. Neurosurgery. 1987;20:222-227.

41. Lechner $H$, Schmidt R, Bertha $G$, Justich $E$, Offenbacher $H$, Schneider $G$. Nuclear magnetic resonance image white matter lesions and risk factors for stroke in normal individuals. Stroke. 1988;19:263-265.

42. Ford CS, Crouse JR, Howard G, Toole JF, Ball MR, Frye J. The role of plasma lipids in carotid bifurcation atherosclerosis. Ann Neurol. 1985;17: 301-303.

43. Meyer JS, Rogers RL, Mortel KF, Judd BW. Hyperlipidemia is a risk factor for decreased cerebral perfusion and stroke. Arch Neurol. 1987;44: 418-422.

44. Dandona $P$, James IM, Newbury PA, Woolard ML, Beckett AG, Cerebral blood flow in diabetes mellitus: evidence of abnormal cerebrovascular reactivity. $B M$ J. 1978;2:325-326.

45. Barnes AJ, Locke P, Scudder PR, Dormandy TL, Dormandy IA, Slack J. Is hyperviscosity a treatable component of diabetic microcirculatory disease? Lancet. 1977;2:789-791.

46. Kee DB, Wood JH. Influence of blood rheology on cerebral circulation. In: Wood $\mathrm{JH}$, ed. Cerebral Blood Flow: Physiologic and Clinical Aspects. New York, NY: McGraw-Hill International Book Co; 1987;10:173-185.

47. Nicolaides AN, Bowers R, Horbourne T, Kidner PH, Besterman EM. Blood viscosity, red-cell flexibility, haematocrit, and plasma-fibrinogen in patients with angina. Lancet. 1977;2:943-945.

48. Thomas DJ, DuBoulay GH, Marshall J, et al. Effect of haematocrit on cerebral blood-flow in man. Lancet. 1977;2:941-943. 